\title{
Practice Pearls for Performing Pleural Ultrasound with Focus on Pleural Effusion and Pleural Thickening
}

\author{
Macarena R Vial ${ }^{1}$ and Horiana B Grosu² \\ 1. Interventional Pulmonology Unit, Clinica Alemana de Santiago-Universidad del Desarrollo, Chile; 2. Department of Pulmonary Medicine, The \\ University of Texas MD Anderson Cancer Center, Houston, TX, US
} for the evaluation of patients with pleural diseases. Ultrasonography is a particularly useful tool in the evaluation of pleural effusion and pleural thickening, with advantages such as portability, low cost, and safety.

\section{Keywords}

Ultrasound, pleural effusion, pleural thickening

Disclosure: Macarena R Vial and Horiana B Grosu have nothing to declare in relation to this article. This article is a short opinion piece and has not been submitted to external peer reviewers. A member of the editorial board reviewed the article before publication.

Authorship: All named authors meet the International Committee of Medical Journal Editors (ICMJE) criteria for authorship of this manuscript, take responsibility for the integrity of the work as a whole, and have given final approval to the version to be published.

open Access: This article is published under the Creative Commons Attribution Noncommercial License, which permits any noncommercial use, distribution, adaptation, and reproduction provided the original author(s) and source are given appropriate credit.

Received: October 16, 2017

Published Online: December 12, 2017

Citation: US Respiratory \& Pulmonary Diseases, 2017;2(1):23-5

Corresponding Author: Horiana B Grosu, Department of Pulmonary Medicine, Unit 1462, The University of Texas MD Anderson Cancer Center, 1515 Holcombe Blvd, Houston, TX 77030, US. E: hbgrosu@mdanderson.org

Support: No funding was received in the publication of this article.

\section{General considerations}

Pleural ultrasound examination can be performed in any position: supine or sitting. A low-frequency phased array transducer of 3.5-5 $\mathrm{MHz}$ is generally preferred for evaluation of pleural diseases. High-frequency linear transducers do not provide acceptable penetration for visualizing deep structures, but do provide better imaging of the pleural lining and better assessment of pleural thickness. The operator should adjust the ultrasound settings such as depth, gain, and time-gain compensation to obtain the best possible image.

\section{Identifying a pleural effusion}

Multiple studies have confirmed that the diagnostic accuracy of ultrasound is superior to that of chest radiography for detection of pleural effusions. ${ }^{1}$ Pleural ultrasound can detect physiologic amounts of pleural fluid and is highly sensitive for effusions of $>100 \mathrm{ml} .^{2}$

Free-flowing effusions accumulate in the most dependent portions of the thorax. Most commonly effusions accumulate in the posterio-lateral costophrenic recesses in seated or supine patients, but they can also accumulate anteriorly in mechanically-ventilated patients in a prone position.

Pleural effusions are best evaluated starting at the level of the diaphragm. The transducer should be placed in a longitudinal plane on the posterior axillary line at the level of the diaphragm with the transducer orientation marker pointed cephalad.

The following structures must be identified to diagnose a pleural effusion: liver, spleen, diaphragm, lung, and chest wall. In the absence of pleural fluid, examination of the costophrenic angle will reveal the diaphragm and underlying organ (liver or spleen), with lung tissue coming into view during deep inspiration; this is called the curtain sign. ${ }^{3}$ This sign, however, does not rule out the presence of loculated or interlobar effusions.

\section{Quantifying pleural fluid}

Several methods have been proposed to quantify the size of pleural effusions, but a precise estimate of fluid volume on ultrasonography is not possible. The largest study of this to date found a strong correlation between calculated and actual volumes drained by thoracentesis. ${ }^{4}$ This uses the formula of: volume (in $\mathrm{ml}$ ) $=16 \times$ parietal-to-visceral pleural distance (in $\mathrm{mm}$ ) at the level of mid-diaphragm to quantify pleural fluid. In general, we measure the deepest pocket of fluid in a still picture, or the number of rib spaces where the fluid can be seen by holding the probe 
Figure 1: Pleural effusion
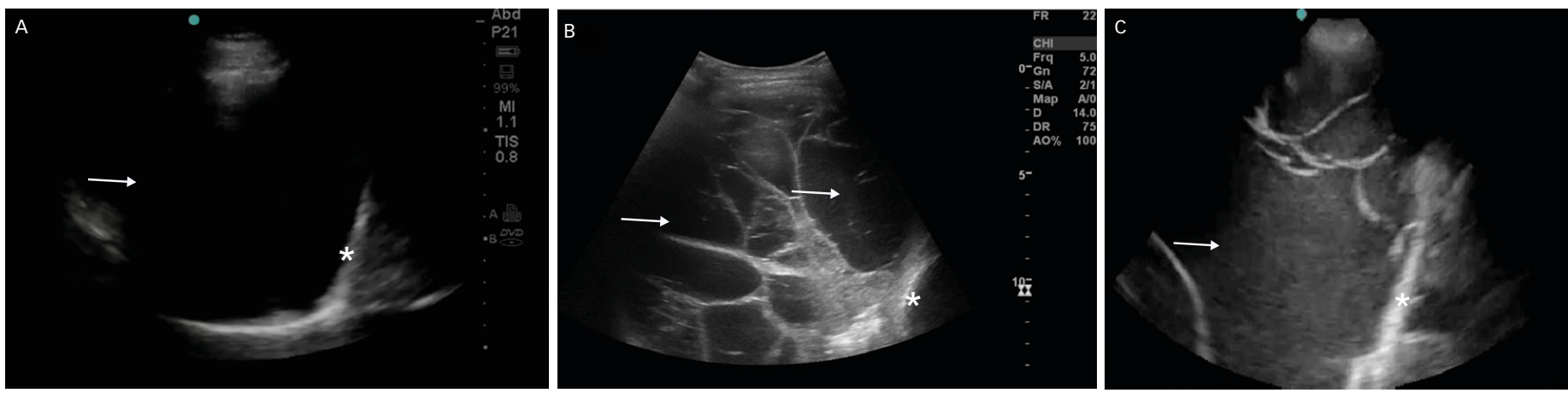

A: Ultrasound image depicting anechoic (black) pleural effusion; B: Ultrasound image depicting an echogenic pleural effusion with multiple loculations in a patient with empyema; c: Ultrasound image depicting homogenous echogenic effusion in a patient with hemothorax. White arrows show locules with fluid and septations. White asterisks show location of the diaphragm.

Figure 2: Pleural thickening
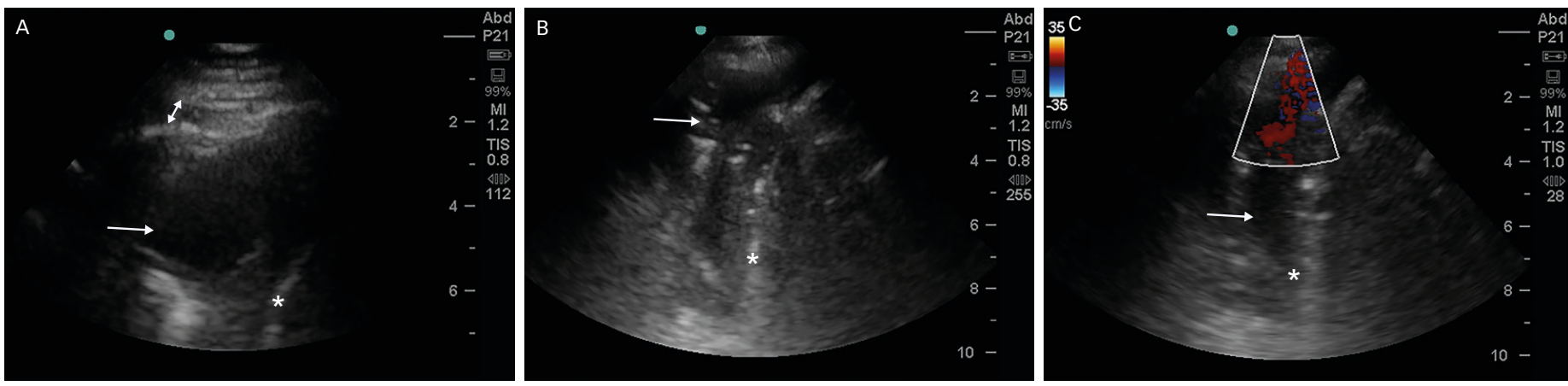

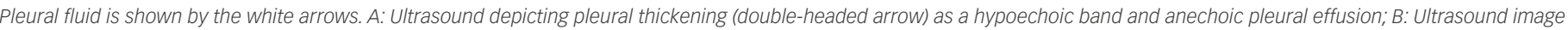
depicting small anechoic pleural effusion; C: Ultrasound image depicting anechoic pleural effusion with Doppler sign. White asterisks show location of the diaphragm.

parallel to the ribs at the diaphragm level and carefully moving it upwards while the operator counts the number of intercostal spaces. This method is also useful for follow up of patients to assess re-accumulation of fluid after an intervention.

\section{Simple versus complex pleural effusion}

simple pleural effusions are anechoic (black) and can be transudative or exudative (Figure 1A). Complex pleural effusions are usually exudative and are subcategorized as homogeneously or heterogeneously echogenic, with or without septations (Figure 1B).

Homogenously echogenic effusions are most often attributable to hemothorax or empyema. (Figure $1 C$ ) The hematocrit sign is the sign in which the high cell count of a hemothorax creates a layering effect in the costophrenic recesses. ${ }^{5}$ Empyema usually has a homogeneously echogenic appearance with multiple loculations. ${ }^{6}$ Heterogeneous echogenic effusion with floating particles that swirl with respiratory movements suggest malignancy.

\section{Pleural thickness}

The normal pleura, with the parietal and visceral layers, measures approximately $0.3 \mathrm{~mm}$ in thickness and can be seen on ultrasound as a 2-mm hyperechoic band representing the pleuropulmonary interphase. It is generally not possible to distinguish between the parietal and visceral pleura on ultrasound, but the visceral pleura moves during respiration in the so-called "sliding sign". Irregularities of the visceral pleura produce comet tail artifacts, which can also be seen in normal conditions, particularly in elderly patients. If there is an increase in pleural fluid, the parietal pleura is seen as a thin echogenic line, sometimes with a deeper hypoechoic layer corresponding to extra pleural fat, but the visceral layer is harder to recognize if the lungs are relatively normal. If there is a partially consolidated or atelectatic lung, the visceral pleura becomes visible as a delicate hyperechoic band in the lining of the lung.

Pleural thickening can be localized or diffuse, and appears as a hypoechoic band (Figure 2A). Diffuse pleural thickening can be seen in inflammatory or malignant disease. Pleural inflammation, particularly chronic inflammation, presents as an irregularity of the pleural line, with some degree of subpleural consolidation. Pleural thickening of $>10 \mathrm{~mm}$, diaphragmatic thickness of $>7 \mathrm{~mm}$, and pleural nodularity are strong predictors of underlying malignancy. ${ }^{8}$

\section{Limitations}

The majority of pleural effusions can be identified using ultrasonography. However, in patients who are obese or have soft tissue edema the image quality may be degraded and difficult to identify when the effusions are highly exudative such as in empyema or hemothorax. In such cases computer tomography may be needed for evaluation and confirmation of pleural effusion. 
In addition, pleural plaques, can be seen as localized hypoechoic lesions, sometimes mimicking a loculated effusion, and color flow Doppler signs can be utilized as adjuncts to routine ultrasonography. A free-flowing pleural effusion will demonstrate flow on color Doppler (Figure 2B and $C$ ). Absence of flow is seen with dense pleural loculations and other abnormalities that can mimic pleural effusions, such as peripheral lung tissue, pleural thickening, or pleural masses.

\section{Conclusion}

Ultrasonography is particularly useful in the assessment of pleural disease. It can identify and characterize pleural effusions and pleural thickening, and provides useful guidance for diagnostic procedures. It is relatively inexpensive, safe, and widely available. However, proper training and continued competency is essential to use this modality and achieve optimal results.
1. Eibenberger KL, Dock WI, Ammann ME, et al., Quantification of pleural effusions: sonography versus radiography, Radiology, 1994;191:681-4.

2. Kalokairinou-Motogna M, Maratou K, Paianid I, et al., Application of color Doppler ultrasound in the study of small pleural effusion Med Ultrason, 2010;12:12-6.

3. Targhetta R, Bourgeois JM, Chavagneux R, et al., Ultrasonographic approach to diagnosing hydropneumothorax Chest, 1992:101:931-4. Usta E, Mustafi M, Ziemer G, Ultrasound estimation of volume of postoperative pleural effusion in cardiac surgery patients, Interact Cardiovasc Thorac Surg, 2010;10:204-7.

5. Mayo PH, Doelken P, Pleural ultrasonography, Clin Chest Med, 2006;27:215-2.

6. Yang PC, Luh KT, Chang DB, et al., Value of sonography in determining the nature of pleural effusion: analysis of 320 cases AJR Am J Roentgenol, 1992;159:29-33.

7. Chian CF SU WL, Soh LH, et al. Echogenic swirling pattern as a predictor of malignant pleural effusions in patients with malignancies, Chest, 2004;126:129-34.

8. Qureshi NR, Rahman NM, Gleeson FV, Thoracic ultrasound in the diagnosis of malignant pleural effusion, Thorax, 2009;64:139-43. 